CONTAMINANT ANALYSIS AUTOMATION DEMONSTRATION PROPOSAL

\author{
M. G. Dodson \\ A. Schur \\ J. G. Heubach
}

October 1993

\author{
Presented at the \\ Human Factors Society Conference \\ October 1993 \\ Seattle, Washington
}

Work supported by

the U.S. Department of Energy

under Contract DE-ACO6-76RLO 1830

\title{
DISCLAIMER
}

This report was prepared as an account of work sponsored by an agency of the United States Government. Neither the United States Government nor any agency thereof, nor any of their employees, makes any warranty, express or implied, or assumes any legal liability or responsibility for the accuracy, completeness, or usefulness of any information, apparatus, product, or process disclosed, or represents that its use would not infringe privately owned rights. Reference herein to any specific commercial product, process, or service by trade name, trademark, manufacturer, or otherwise does not necessarily constitute or imply its endorsement, recommendation, or favoring by the United States Government or any agency thereof. The views and opinions of authors expressed herein do not necessarily state or reflect those of the United States Government or any agency thereof. 
CAA Demonstration Proposal

\title{
Contaminant Analysis Automation Demonstration Proposal
}

\author{
Abstract \\ Mike Dodson \\ Anne Schur \\ Janet G. Heubach
}

The nation-wide and global need for envirnnmental restoration and waste remediation (ER \&WR) presents significant challenges to t: a analytical chemistry laboratory. The expansion of ER \& WR programs forces an increase in the volume of samples processed and the demand for analysis data. To handle this expanding volume, productivity must be increased. However. The need for significantly increased productivity, faces contaminant analysis process which is costy in time, labor. equipment. and safety protection. Laboratory automation offers a cost effective approach to meeting current and future contaminant analytical laboratory needs.

The proposed demonstration will present a proof-of-concept automated laboratory conducting varied sample preparations. This automated process also highlights a graphical user interface that provides supervisory control and monitoring of the automated process. The demonstration provides affirming answers to the following questions about laboratory automation:

Can preparation of contaminants be successfully automated?

Can a full-scale working proof-oi-concept automated laboratory be developed that is capable of preparing contaminant and hazardous chemical samples? Can the automated processes be seamlessly integrated and controlled? Can the automated laboratory be customized through readily convertible design? Can automated sample preparation concepts be extended to the other phases of the sample analysis process?

To fully reap the benefits of automation. four human factors areas should be studied and the outputs used to increase the efficiency of laboratory automation. These areas include: 1) laboratory configuration.

2) procedures. 3) receptacles and fixtures. and 4) human-computer interface for the full automated system and complex laboratony information management systems. 
CAA Demonstration Proposal

\section{Contaminant Analysis Automation Demonstration Proposal}

\subsection{Purpose}

It is the purpose of this demonstration to explain the capabilities and human factors of an automated contaminant analysis laboratory. The specific objectives of the demonstration are:

- to show that contaminantpreparation processes can be successfully automated

- to present a working proof-of-concept automated analytical laboratory capable of timely and safely preparing of contaminant and hazardous samples

- to show that different analytical processes can be seamlessly integrated and controlled

- to demonstrate that an automated laboratory can be customized through readily convertible design

to show that automated sample preparation concepts are extensible to the other phases of the sample analysis process.

The proposed demonstration will present a proof-of-concept automated laboratory conducting varied sample preparations. This automated process also highlights a graphical user interface that provides supervisory control and monltoring of the automated process.

\section{Introduction}

The effort to restore the environment and remediate waste across the nation, in general, and at The Department of Energy (DOE) siles, in particular, presents a number of challenges to analytical laboratories. An accumulation of 50 years of radioactive and hazardous wastes are stored and buried at numerous DOE sites across the United States. The accumulated wastes located at each of the sites must be characterized prior to remediating the waste and restoring the environment. However, the historical manifests of the contents of these wastes can be unknown, incomplete, and/or inaccurate. The analytical chemistry sample load required for ER\&WM is forecast to increase from 50 to 100 fold within the next seven years and to remain at that level for 10 to 30 years. Sample analysis is the mechanism by which the contaminated wastes are analyzed for element, isotope, and compound content. The analytical process consists of three phases: sample preparation, sample analysis, data interpretation and reporting.

Standard analytical chemistry analyses are labor intensive operations utilizing costiy tiaired staff, sophisticated instrumentation, and expensive facilities. Using current analytical chemistry methodologies, staffing and facilities, it is projected that the shear ichume $r$ '. the DOE sites will exceed the capabilities of qualified DOE, federal and commers:al da to... dtories in this country. Within this scenario a DOE funded program working to att 7 . ' $i$ i he aulsmatis 2 of high use analyses has begun to develop new methods and machines to $1 \therefore \mathrm{p}$, olve the jrot ilem. Laboratory automation can help relieve the pressure from the increased s a th voism ${ }^{\circ}$. Indeed. recent market studies forecast automated systems growth for the UCEE se ter to be $\$ 2.8$ billion over the next ten years.

The hazardous nature of the samples and re-agents represent another driv'ng in developing automated contaminant analysis technology. Many of the mancit. procedures for analyzing the contaminated materiais and re-agents are labor intensive reculuing frequent and direction manipulations by laboratory staff. Sample preparation typically involves numerous operations. e.g. . poring. mixing. grinding. digesting solids, weighing, adjusting pH, 
fusing. emulsifying, etc. Laboratory automation technology offers one approach to meeting current and projected contaminant analysis needs. Analytical automation technology provides a means to: 1) increase throughput and the processing accuracy, 2) reduce the interpretation time of the collected data, 3) reduce the potential for unacceptable personnel exposure to hazardous substances, and 4) allow analytical processes to be adjusted to the unique characteristics of the contaminated samples.

\subsection{The Contaminant Analysis Automation Program}

The Contaminant Analysis Automation (CAA) program began in 1989*. Pacific Northwest Laboratory at the Hanford DOE site in Washington state has beer a partner with other national laboratories in this project from its inception. Since the beginning of the program, a series of new technologies have been developed and passed to the private sector for commercialization. In addition to the development of new technology, a variety of automation issues emerged which may define basic design factors for future automated laboratories and require extensive human factors research and development work. Five areas were identified that will be most impacted:

the configuration of the laboratory itself

the receptacles or fixtures used to contain the samples while they are transported. prepared, and analyzed

the role of personnel who perform the sample analysis.

the management of laboratory information.

automated sample preparation concepts are extensible to the other phases of the sample analysis process

Automated laboratories offer a significant departure from traditional laboratory design. In contrast, an observer taken into most existing analytical laboratories will be struck by the similarity of the laboratory to the laboratories they experienced in high school and college. Indeed. most analytical chemistry laboratories conceptually replicate the laboratories used by Thomas Edison. While many changes in laboratory design have occurred, most are changes in construction materials (epoxy resin instead of shiny black paint), services delivery (gases. air. water), cabinetry, and protection provisions (exhaust engineering). Automated analytical laboratories change the basic operation of the laboratory and its personnel.

\subsection{Sample Analysis Practices}

Laboratory automation will be place chemists in new supervisory and human-technology collaborative roles in the laboratory. Communication and co-ordination between the human and the automated systems are critical for safe, efficient, and accurate operation. A usercentered system design approach was adopted within a systems engineering framework throughout the CAA program.

CAA Program funded under contract to the Department of Energy. 
Chemists currently perform many analysis processes manually following very specific procedures. The task analysis revealed the sample process to be comprised of eight distinct operational activities.

1. Instrument preparation

2. Sample preparation

3. Calibration verification

4. Track samples

5. Data collection

6. Clean-up

7. Documentation

8. Data reporting

Standard laboratory practices are time consuming, repetitive, often tedious, require the laboratory staff to possess a diverse range of skills and knowledge, and expose chemists and technical laboratory staff to potential hazards. Table 1 characterizes the tasks as they have been traditionally performed and qualitatively compares the manual and automated performance of each task characterization. The accuracy to which each step is performed is critical to sample preparation.

TABLE 1. The Characterization of Sample Preparation Tasks

Task Description

Repetitive

Tedious

Time consuming

Skill required

Increased sample volume

Safety \& exposure

\section{Manual}

Varied accuracy

Varied consistency

Varied accuracy

Varied Consistency

Limited by staffing

Varied

Increased workload induces error

Potential human
Automation

Accurate \& consistent

Accurate \& consistent

Faster than human

Constant level

Accommodates sample increase

Decreased exposure potential

The task analysis provided the framework for defining user requirements and to identify the essential information required by the user to perform the identified tasks. Users identified the sample preparation process and data interpretation as the two principle bottlenecks of sample analysis. However, users identified the sample preparation as more critical process.

Therefore. sample preparaticr. received first priority. Typical tasks or operations that occur within sample preparation were identified and categorized into three groups; tasks which get and place items (receptacles, liquids, etc.), tasks which perform actions on an ttem or specify the degree to which an action should occur (heat, remove, mix. etc.), and tasks which involve the recording of information (weight, time, etc.). 
Program efforts focused further on the development of an human-computer interface (HCI) that would support the automated preparation of samples. Within the automated domain there are two critical operational areas: the ability to develop procedures by which the samples are prepared and the ability to "train" or teach the robot the location of the workcells which will perform the sample preparation work.

\subsection{Automating Contaminant Sample Analysis}

Robotics and advanced computer software techniques will be used to perform sample analysis. while neural networks and knowledge-based systems will provide improved quality of the evaluation of the analysis results. Collectively they will be integrated to form an intelligent workbench. (Figure 1).

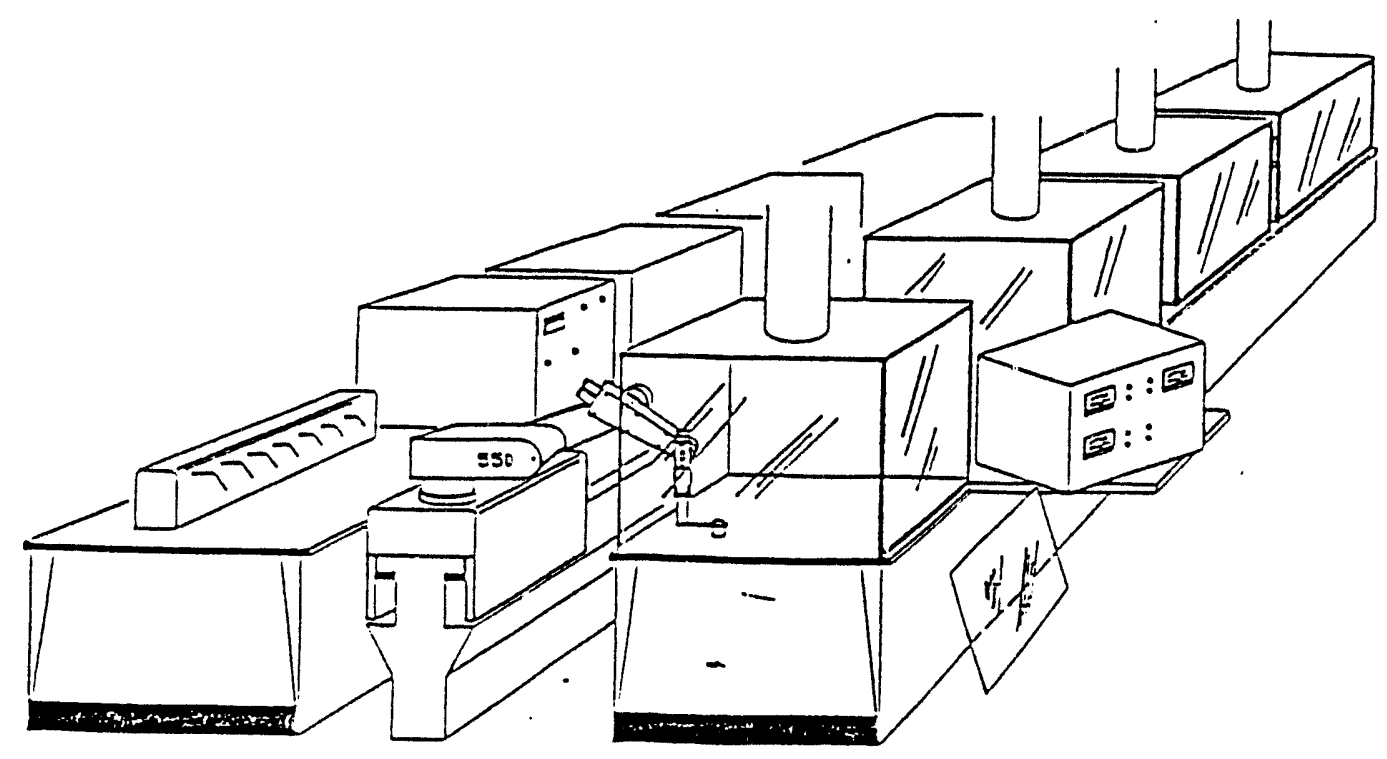

Figure 1. Intelligent Workbench

The sample processing design supports the following user functions: sample analysis set-up. operational readiness, process run, data collection, status reporting, scheduling, and $\mathrm{QA} / \mathrm{QC}$ documentation. Table 2 summarizes the automated functions.

Control of the tasks will be performed by directly interfacing all cell equipment, robot and instruments, to a dedicaled computer and communicating to these system components via a human-computer interface. 
TABLE 2. Expected Automated Functions

Function

Sample analysis Set-up

Operational readiness

Process run

Status Reporting

Scheduling

Documentation
Description

Specify robot locations, self-calibration, select create procedures

Verify sufficient resources, safety verification review and acknowledge each procedure step

Start sample analysis, pause run, sample status, preview collected data, look ahead

System process, samples

Maximum sample throughput, resource optimization

Standards and guidelines, report generation, plot generation. on-line assistance

\subsection{Conclusions and Human Factors Research Applications}

Automation techniques offer a cost effective and safe approach to meeting current and future analytical laboratory needs if properly designed and implemented. The Contaminated Analysis Automation demonstrates a working proof-of-concept which shows that:

\footnotetext{
. contaminant sample preparation processes can be successfully automated

. automated analytical laboratories are capable of timely and safely analyzing contaminant and hazardous chemical samples different analytical processes can be integrated and controlled automated laboratories can be customized through convertible design automated sample preparation concepts are extensible to the other phases of the sample analysis process
}

To fully reap the benefits of automation the following human factors areas should be studied and the outputs used to increase the benefits of laboratory automation.

\section{Laboratory Configuration}

The proposed intelligent workbench is a departure from existing automated platforms and current laboratory facility configurations. As automated systems are generated and migrate into laboratories the current configurations will be strained, if not unusable. Two different situations will require focused attention. First, there will be a tendency to make as little change as posstble to existing facilities while trying to incorporate new equipment and way of performing work. Second. the design of new facilities where a clean slate approach is adopted will conflict with the status quo. In both, there will be a need to create new ways of looking at the space within the laboratory. Modularity and convertability will be required. 


\section{Procedures}

Automated procedures currently emulate manual processes. However, automation techniques, by their nature. should not require manual process emulation. The impact of automation on manually performed EPA procedures should be identified to further streamline and adjust the sample preparation process.

\section{Receptacles/Fixtures}

The majority of the receptacles used to prepare samples are made of glass. These fixtures are unsuited, in most instances. for use in the automated environment due their durability. In addition, current laboratory glassware varies significantly in size and shape. Developing fixtures suited to the automated laboratory environment would be more cost effective than adapting the robot to existing fixture design. Indeed, research and development work on fixture design would benefit non-automated laboratories as well, e.g., packaging and storage.

\section{Human-Computer Interface Development}

To be effective, laboratory automation requires a fully developed $\mathrm{HCI}$ to support the entire sample analysis process. sample preparation, and data interpretation. Supervisory control and monitoring capabilities should also be extended to include the ability to perform status monitoring. In addition. complex data handling systems or Laboratory Information Management Systems (LIMS) will be required to collect all sample data from sample entry into the laboratory. through analysis. during sample tracking, and through data reduction and report preparation. Design of the interfaces of such LIMS must be focused on the reference experiences of the analytical laboratory personnel.

\subsection{Selected References}

NRTDP Five Year Plan. Contaminant Analysis Automation Section. Draft August 15, 1990.

Gaines. B.R. (1981). A conceptual Framework for Person-Computer Interaction in Complex System. Transactions on System, Man, and Cybernetics. 18 (4), 532-541.

Groover. M.P., Weiss. M... Magel, R.N., Odrey, G. (1986) Industrial Robots Technology. Programming and Applicalions. McGraw-Hill.

Hurst, J.J., Mortimer, J.W. (1987). Laboratory Robotics: A Guide to Planning Programming, and Applications. VVCH Publishers.

Isenhour. T.L.. Eckert. S.E. (1989). Intelligent Robots - The Next STep in Laboratory Automation. Analvtical Chemistry, 61 (13).

Schneiderman, B.. (1983). Direct Manipulation: A Step Beyond Programming languages. Computer. 16 (89). 57-69.

Settle. J.R. Diamondstone. B.l., Kingston, H.M. (1989). An Expert - Database System for Sample Preparation by Microwave Dissolution. 1. Selection of Analytical Descriptors. Joumal of Chemistn information Computer Sciences. 29 (1). 

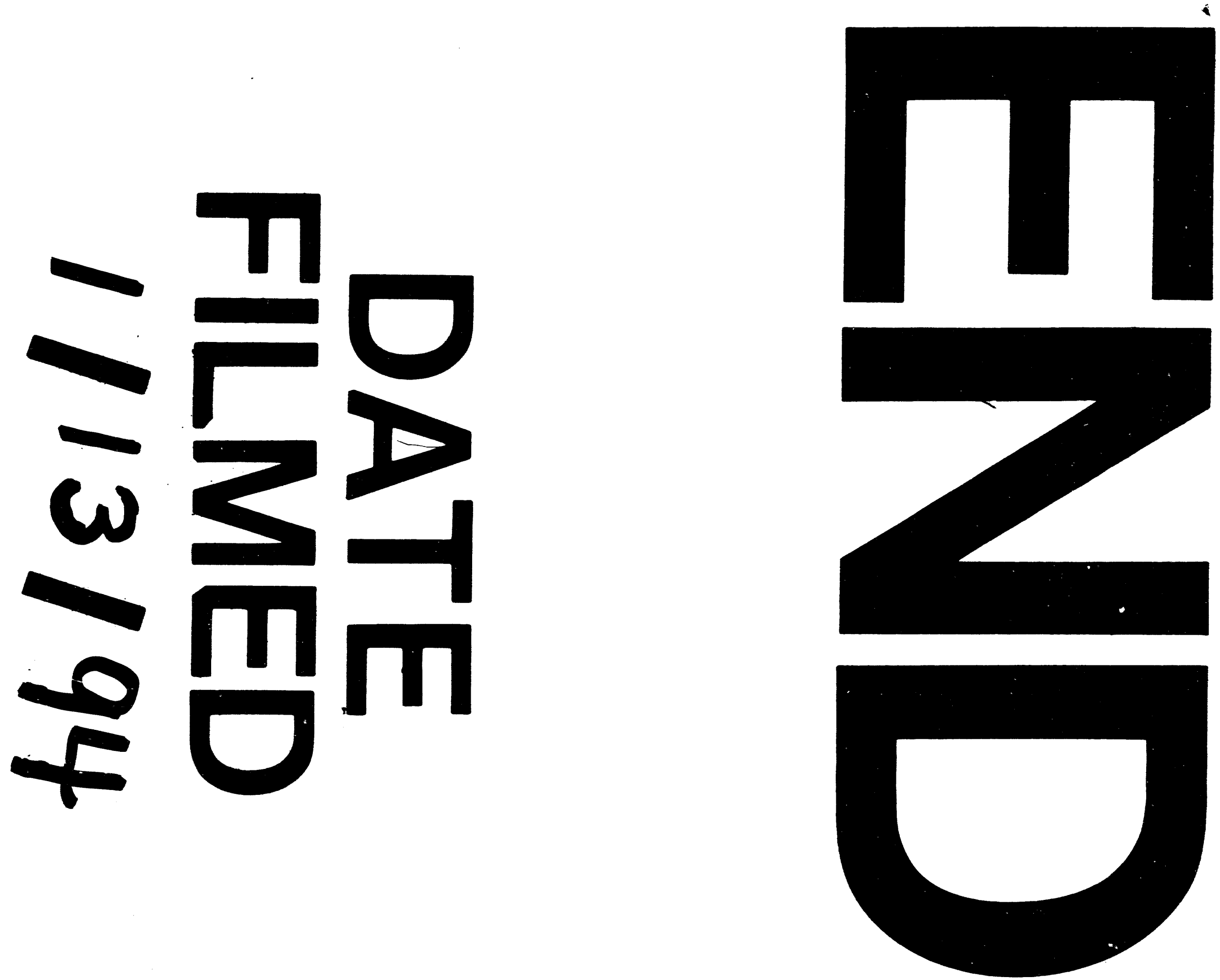
